DOI: https://doi.org/10.33103/uot.ijccce.20.4.7

\title{
The Effect of Mode Area and Refractive Index for Optical TE Mode Propagation in Hybrid LN/Si Electro-Optic Structure of Mach-Zehnder Modulator
}

\author{
Luma Z Mohammed ${ }^{1}$, Makram A. Fakhri² , A. K. Abass ${ }^{3}$ \\ ${ }^{1,2,3}$ Laser and Optoelectronic department, University of Technology, 10066 Baghdad, Iraq. \\ lumazuhair@gmail.com,mokaram_76@yahoo.com,140017@uotechnology.edu.iq
}

\begin{abstract}
We propose and analyse a silicon based hybrid modulator on the nano thin film of the lithium niobate or commonly known as silicon-on-insulator technology. The Mach-Zehnder stripe optical waveguide of electro-optical modulator operats at $\mathrm{GHz}$ frequencies with large bandwidth and low losses between electrical and optical frequencies.The design and simulation of MachZehnder modulator is based on a hybrid integration platform of silicon and lithium niobate that satisfies a single mode condition. The Silicon stripe waveguide is of $0.6 \mu \mathrm{m}$ thickness in a silicon on insulator (SOI) of width $15 \mathrm{um}$ and 0.05 um thickness $x$-cut LiNbO3 thin film, all sets use the pulse laser deposition (PLD) method. The Optical electric field distributions and effective mode area in the optical-waveguides were studied and discussed in this designated waveguide.The relationship between the width of waveguides regions with effective mode index and effective mode area was investigated. At 0.6 um width of waveguide and 0.2 um thickness, the effective mode index 1.9802 was recorded while the effective mode area 0.144 um2 was monitored. This shows the decrement in both: the width and thickness of the waveguide with the effective mode index and effective mode area.
\end{abstract}

Index Terms—lithium niobate; effective mode index; effective mode area; thin film; TE Mode

\section{INTRODUCTION}

External electro-optic modulators based on lithium niobate (LN) are key fundamental components in modern communications, and microwave-photonic systems that convert high-speed radio-frequency (RF) signal into the optical domain. This is important for optical switching, generation of high-bandwidth signal, data communication, waveform shaping , ranging and timing in RF photonics, sampling and measurements of ultrafast signals $[1,2]$.

Enormous efforts have been made to releasing high performance optical modulators with different material platforms such as silicon. However, the submicrometer spatial confinement of light and dispersion effect of the free carrier leads to absorption losses and nonlinear voltage response. The signal may be distorted due to the intrinsically absorptive and nonlinear dispersive of the free-carrier [4, 5].

Another preferred material, lithium niobate (LiNbO3), is an excellent uniaxial crystal material due to its nonlinear optical characteristics [3] that capable of wave-guiding in the modulators. The $\mathrm{LN}$ has high value of the electro-optics coefficient around $30 \mathrm{pm} / \mathrm{V}[4$, 
5], good piezoelectric [6], pyroelectric [7] and photo-elastic [2, 6] properties, strong Pockels [8] and photorefractive (PR) [9] effects. wide optical bandgap [10], and good temperature stability [4]. The LN is suitable for optical waveguide applications [11], zerochirp transmission systems of the fiber-optic [9, 12], lasers Q-switches [13], long-distance optical communication [13], analog optical links [14], and the optical filters reconfigurable [6] systems.

The Hybrid silicon/LN material system that assoiates the scalability of silicon photonics with LN produces an excellent modulation [4] and structure that widely used in EOMs which is known as phase modulator [15], and also deploys in Mach-Zehnder modulator (MZM). A basic confront in the production of MZM modulators is the fabrication of high-quality waveguide structures. The fabrication of waveguides has been progressed using a few techniques including dry-etching[16], proton exchange(PE) [17], pulse laser deposition(PLD) [11, 18] and chemo-mechanical polishing [19].

In this paper, we simulate and analyze the heterogeneous silicon/LN material system for EOM using COMSOL MULTIPHYSICS [20]. It is used to design and model a strip waveguide structure with optimization for low propagation and coupling loss. The study engages single mode propagation along with its corresponding effective mode index and effective mode area in respect to the height and width of silicon waveguide. The design at wavelength $1.55 \mathrm{um}$ uses the TE-polarized fundamental guided mode, which is also used in conventional silicon photonics[3].

\section{DEVICE DESIGN AND METHOD}

The schematic view of the heterogeneous silicon/LN configuration of silicon-on-insulator (SOI) based MZM employs electro-optic effects as shown in Fig. 1. The input power is divided evenly into two guided wavelengths that come out from the first directional coupler[1].Both of the waveguides form the two MZI arms as depicted in Fig 1(a). The Inverted electric fields were applied to both arms to modify the values of the refractive indices, hence led to the phase shift $\Delta \varphi$ typically between $\pi / 2$ and 0 radians.[21]. This phase shift is opposite in phase for the two arms [22]. The length of interaction between the waves of the light and the RF electric field modulation can be varied accordingly. The travelling wave is applied in MZM structures for many applications such as the TE-polarized fundamental guided mode of waveguides[23].In our design, we considered device fabrication in the hybrid Si-LN optical mode with LN thin film of 0.2 um thick. The $x$-cut $\mathrm{LN}$ in the hybrid system stands bonded on the top of various thicknesses of silicon oxide $\mathrm{SiO}_{2}$ (cladding material) layer was deposited on silicon substrate [2] as shown in Fig. 1(b) using pulse laser deposition technique (PLD) [18]. The LNthin film on the top of a highindex contrast silicon photonic (core material) waveguides is intended for two reasons. Firstly, to act as part of the upper cladding for the modes of the optical waveguide [24] and secondly to associate the scalability of LN for maximum modulation [4]. The Thin deposited gold was use as a travelling wave electrodes [25] on top of the structure in order to get impedance-matching and high-bandwidth performance [26].

The Single mode conditions of waveguide were explored using Y-branch design. The layer of the deposited silicon is to control the pathed light, both of the bound zone outside and also under the region of the bonded LN [2] In optimizing the structure, the stripe waveguide thickness was varied from $0.145 \mathrm{um}$ to $0.2 \mathrm{um}$. The width of silicon waveguide that falls beyond this range was not examined because it did not support single mode operation [27]. An effective and simple technique was used to solve the distortion loss and 
the distribution of the mode. The waveguide geometry was meshed in different algorithm whereby this algorithm has the ability to adapt to change with the structure of the optical waveguide. After meshing the structure of the waveguide, the Maxwell's equations were transformed into the Eigen value matrix problems. This was solved by the techniques of the sparse matrix to find the mode indices effective and the modes optical waveguide distribution [28].

(a)

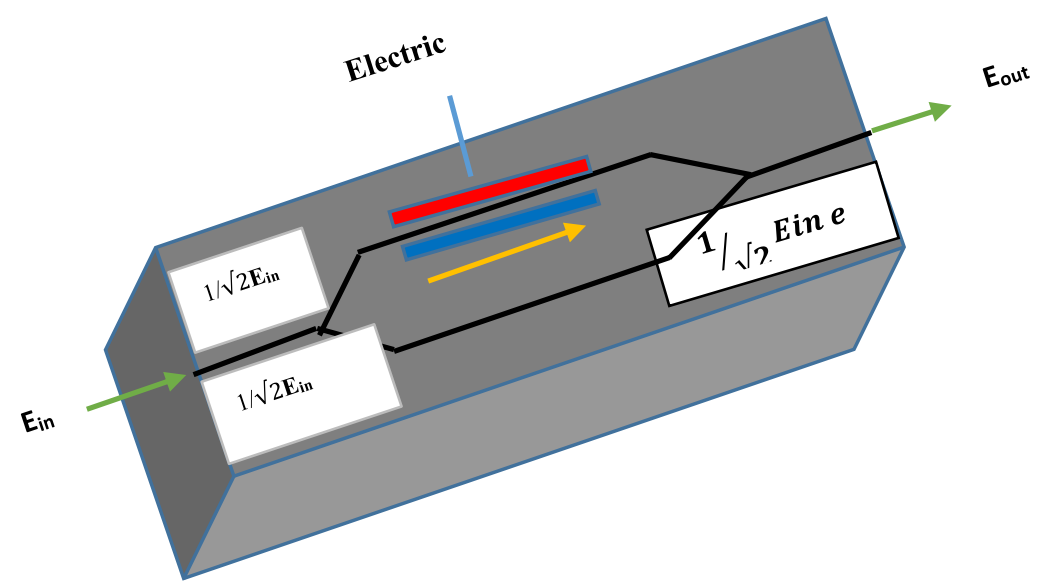

$\mathbf{G}$

G

(b)

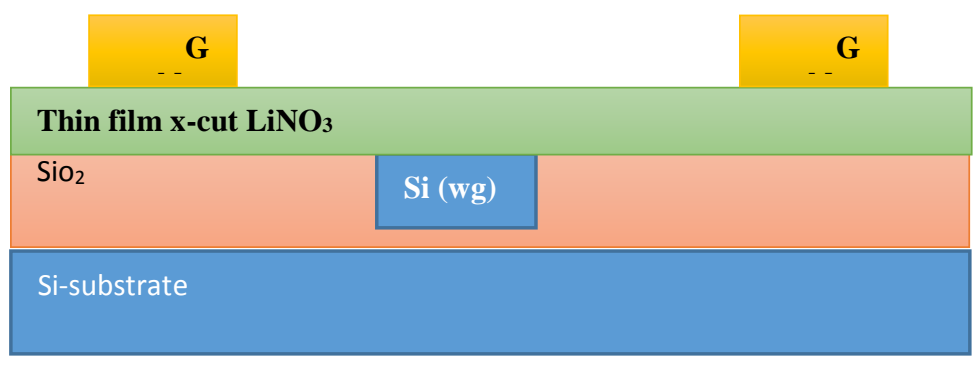

Fig. 1: (A) 3D SCHEMATIC DIAGRAM OF HYBRID OF SILICON/LN ELECTRO-OPTIC MACH-ZEHNDER MODULATOR (B) CROSSSECTIONAL SCHEMATIC OF THE MZM AND WAVEGUIDE'S CHANNEL. (NOTE: IMAGE IS NOT UP TO SCALE.)

\section{A-Simulation Results}

\section{DESIGN PARAMETERS}

In order to have a single mode condition and enhance the overall Pockels modulation effect, we simulated the waveguide structure with width between $0.6 \mathrm{um}$ and $1 \mathrm{um}$. This is to prevent the signal distortion when light makes its transition and to ensure much confinement of the mode in the wave guide region that causes overlapping integral of optical field and the electrostatic field distribution as shown in Figure 2. The optical properties were controlled with an applied electrical signal. The Pockels effect comes to play when applying, the electric field changes the phase of the wave that passes through the crystal. Eventually, this led to changes in the refractive index of $n_{\mathrm{Si}}(3.48)$ and $\mathrm{n}_{\mathrm{LN}}(2.2)$ at wavelength 1.55um [1] since the waveguide index is higher than LN thin film that gives the index which is different in capability to control the mode size and location. The Optical simulation effective index for the TE-guided mode is 2.0653 at thickness $0.2 \mathrm{um}$ as presented in Figures. 2 (a) \& (b). The field distortion of the waveguide at thickness 0.145 um is shown in Figures 2 (c) \& (d). The low value of the optical index between the effective materials of the $\mathrm{LiNbO} 3$ and the Si prevents coupling of the optical modes with the handle. The effective refractive index 1.9802 was observed in $\mathrm{Si}$. The Low values of dielectric constants will be decisive in designing the radio frequency for future high-frequency devices[4]. 


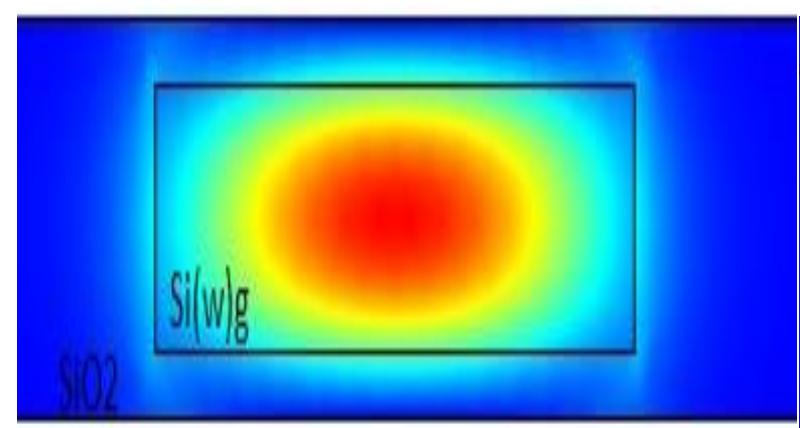

(a)

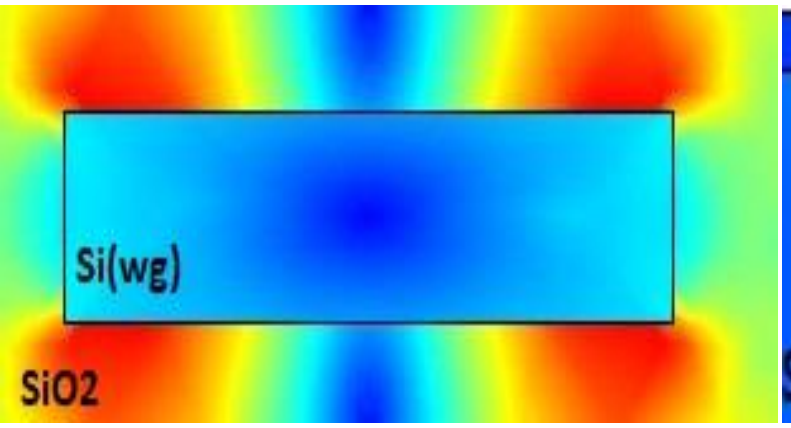

(c)

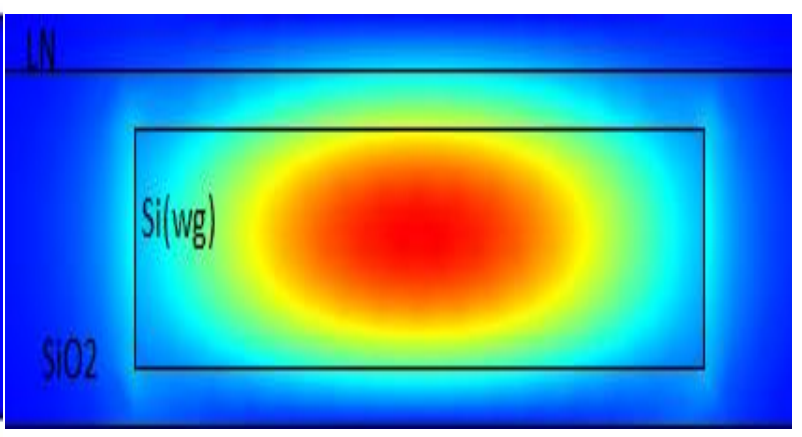

(b)

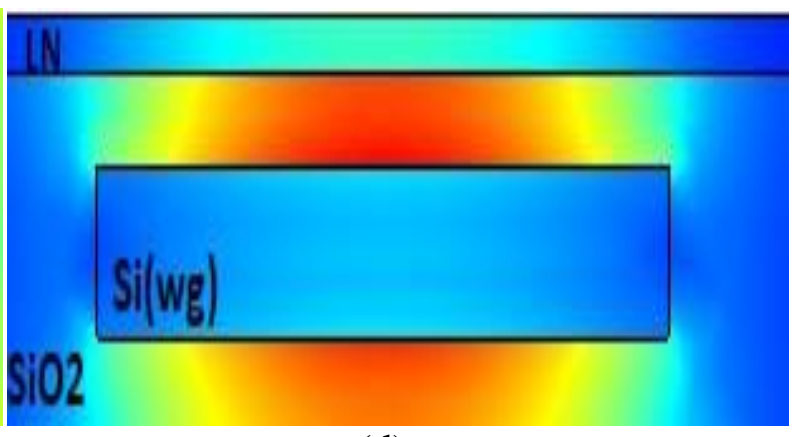

(d)

FIG. 2 (A) AND (B) INTENSITY OF E- FIELD DISTRIBUTIONS FOR THE FIRST MODE INSIDE WAVEGUIDES WITHOUT LN LAYERS AND, (C) AND (D) WITH LN LAYERS

The selection of the width and thickness of the silicon waveguide was considered for vanishing the high order modes and satisfying single mode condition as illustrated in Figure 3(a). The effective refractive index decreases with the thickness and width. The effect of LN thin film is shown in Figure 3(b), which record a high effective index variation with the waveguides width. Therefore, it is better to have wider waveguides to reach the ultimate modulation efficiency [3].

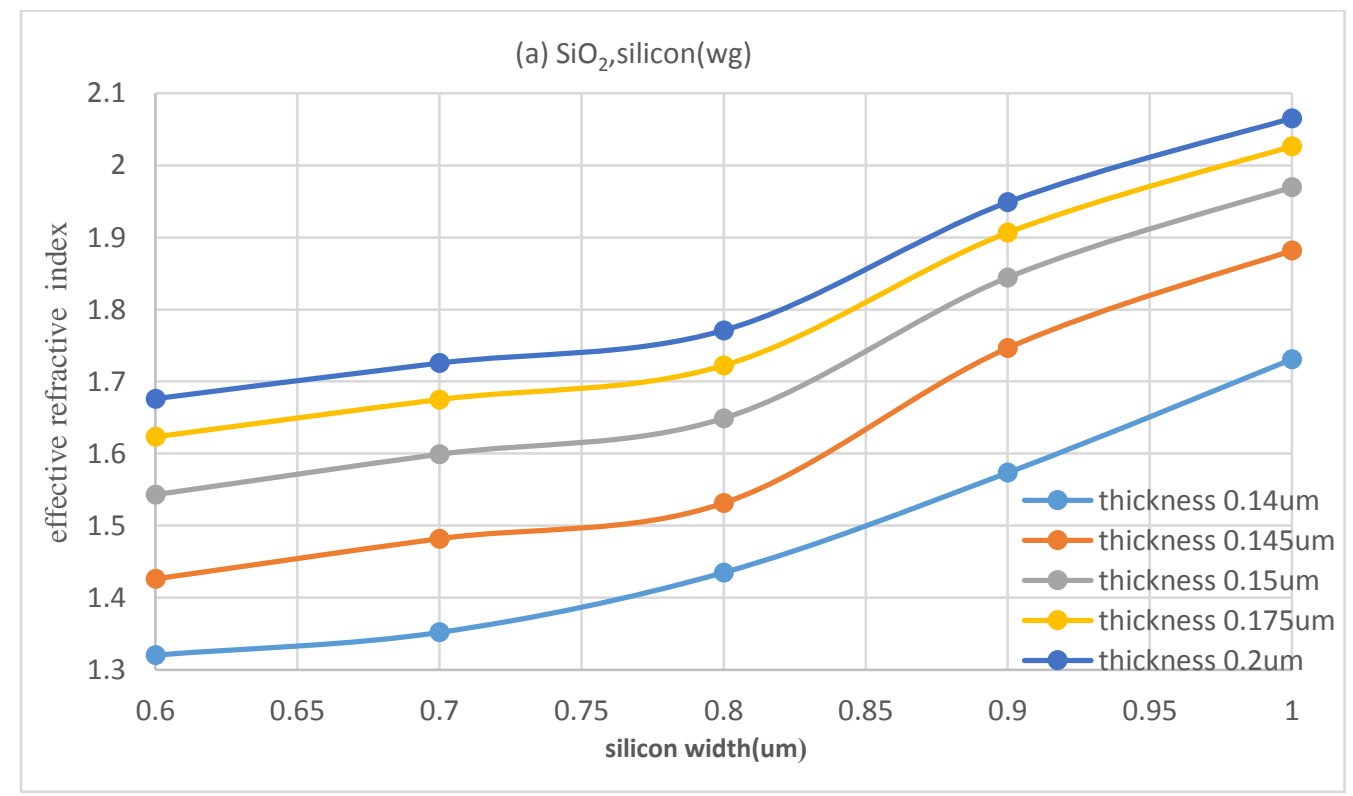




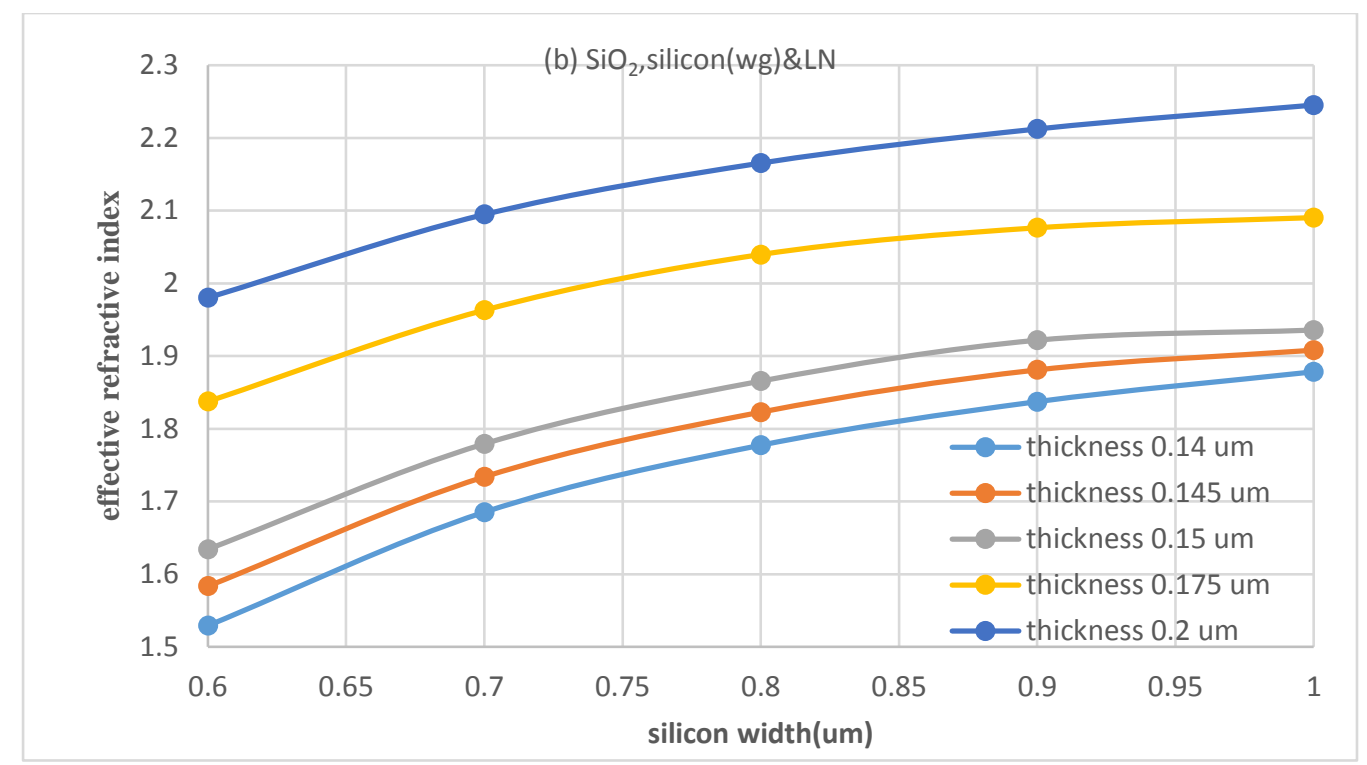

FIG. 3(A,B) EFFECTIVE REFRACTIVE INDEX OF ELECTRIC MODE(TE) MODE VERSE WIDTH OF Si WAVEGUIDE FOR DIFFERENT THICKNESS WITH AND WITHOUT LINBO3 THIN FILM LAYER

Figure 4 depicts the relationship between the effective mode area and the waveguide width. The minimum value of the mode size at a specific width is around 0.9 um after bonding the $\mathrm{LN}$ layer to $\mathrm{SiO}_{2}$. The areas of the small effective mode are usually caused by a strong guiding, where the bend losses and other effects of external disturbances are weak.

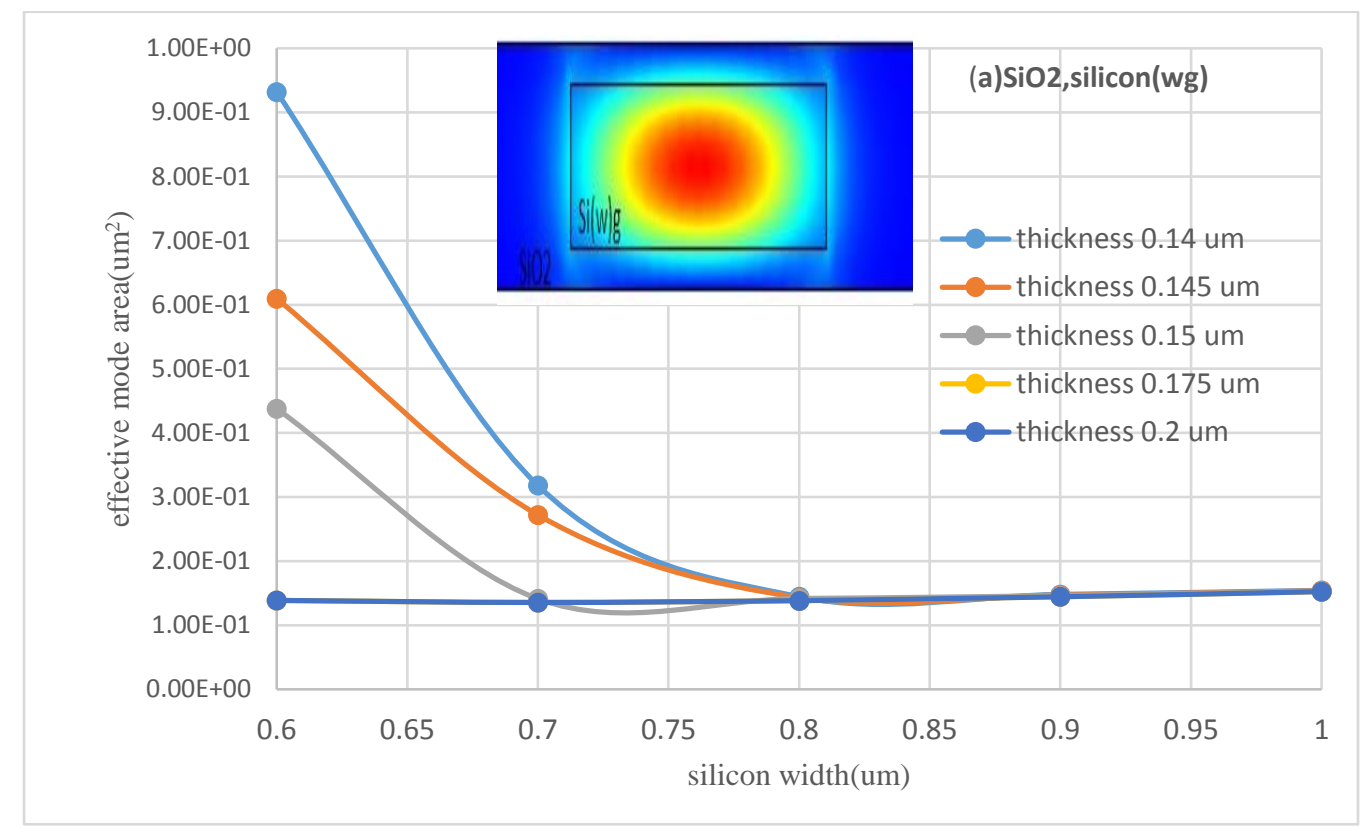

(a) 


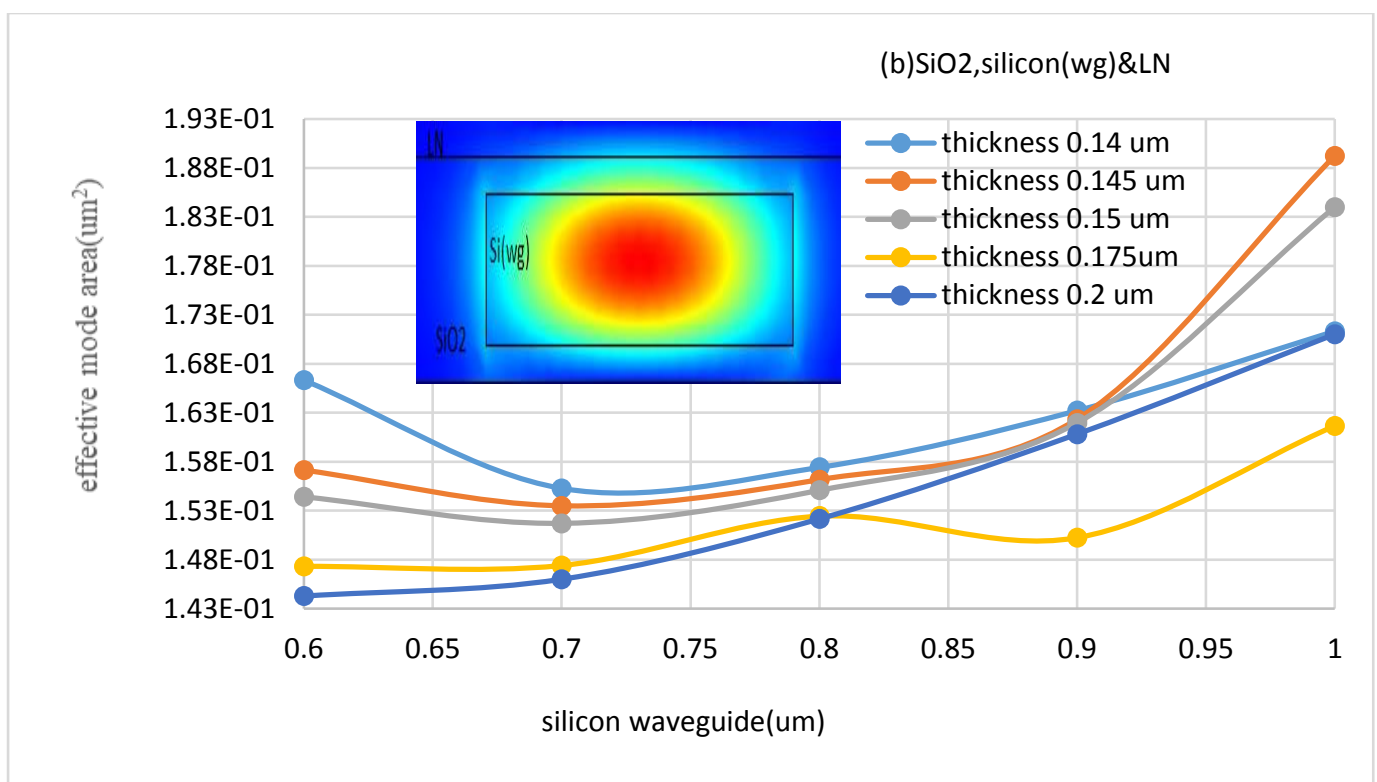

(b)

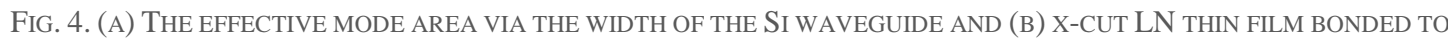
SIO2 LAYER

It is noted that when the width increases, the region of the waveguide expands and the effective mode area increases. As a result, the decrement of waveguide width gives a good light confinement inside it, also resulting in a small mode size. In the inset of Fig. 4(b), for $0.2 \mu \mathrm{m}$ layer and $0.6 \mu \mathrm{m}$ wide channel waveguide, the effective mode area was small in size as $1.44 \mu \mathrm{m} 2$ (product of $1 / \mathrm{e}$ intensity in the vertical and the horizontal directions).

\section{IV.CONCLUSION}

The simulation of the optical transverse-electric (TE) mode considering waveguide width or mode-shape modulation was successfully implemented as a way to obtain a single mode matching in the waveguide. The single-mode condition was satisfied at width $0.6 \mathrm{um}$, the thickness of $\mathrm{x}$-cut $\mathrm{LiNbO}_{3}$ thin film layer $0.05 \mathrm{um}$ and the $\mathrm{SiO} 2$ clad structure of $0.3 \mathrm{um}$. The $\mathrm{Si}$ waveguides thickness was selected as 0.2 um in our simulations that gave strongly a confine light,effective mode area $0.144 \mu \mathrm{m} 2$, and 1.9802 effective refractive index. The relationship between the width of waveguides regions with effective mode index and effective mode area demonstrated and indicated the decrement in both of the width and thickness of the waveguide which resulted in lowering the effective mode index and effective mode area

\section{REFERENCES}

[1]. O. Weigel et al., "Bonded thin film lithium niobate modulator on a silicon photonics platform exceeding 100 GHz 3-dB electrical modulation bandwidth," vol. 26, no. 18, pp. 23728-23739, 2018.

[2] L. Chen, "Hybrid Silicon and Lithium Niobate Integrated Photonics," The Ohio State University, 2015.

[3] HH. Han, B. Xiang, T. Lin, G. Chai, and S. J. C. Ruan, "Design and Optimization of Proton Exchanged Integrated Electro-Optic Modulators in X-Cut Lithium Niobate Thin Film," vol. 9, no. 11, p. 549, 2019.

[4] M. Xu et al., "Michelson interferometer modulator based on hybrid silicon and lithium niobate platform," vol. 4, no. 10, p. 100802, 2019.

[5].S. Germer, "Design and analysis of integrated waveguide structures and their coupling to silicon-based light emitters," 2015.

[6]A. Rao and S. J. I. J. o. S. T. i. Q. E. Fathpour, "Heterogeneous thin-film lithium niobate integrated photonics for electrooptics and nonlinear optics," vol. 24, no. 6, pp. 1-12, 2018. 
[7]Y. Al-Douri, M. A. Fakhri, A. Bouhemadou, R. Khenata, and M. Ameri, "Stirrer time effect on optical properties of nanophotonic LiNbO3," Materials Chemistry and Physics, vol. 203, pp. 243-248, 2018.

[8].A. Rao, "Thin-film Lithium Niobate Photonics for Electro-optics, Nonlinear Optics, and Quantum Optics on Silicon," 2018.

[9].M. R. d. R. Gesualdi, C. Jacinto, T. Catunda, M. Muramatsu, and V. J. A. P. B. Pilla, "Thermal lens spectrometry in pyroelectric lithium niobate crystals," vol. 93, no. 4, p. 879, 2008.

[10]. A. Mohamedelhassan, "Fabrication of Ridge Waveguides in Lithium Niobate," ed, 2012.

[11].S. Taleb, M. Fakhri, and S. J. J. o. O. R. V. Adnan, "PHYSICAL INVESTIGATIONS OF NANOPHOTONIC LiNbO3 FILMS FOR PHOTONIC APPLICATIONS," vol. 15, no. 4, pp. 261-269, 2019.

[12].A. J. Mercante, D. L. Eng, M. Konkol, P. Yao, S. Shi, and D. W. J. O. I. Prather, "Thin LiNbO 3 on insulator electro-optic modulator," vol. 41, no. 5, pp. 867-869, 2016.

[13].M. A. Fakhri, Y. Al-Douri, U. Hashim, E. T. Salim, D. Prakash, and K. J. A. P. B. Verma, "Optical investigation of nanophotonic lithium niobate-based optical waveguide," vol. 121, no. 1, pp. 107-116, 2015.

[14].S. Pal and S. J. O. C. Gupta, "Junction-less optical phase shifter loaded silicon Mach-Zehnder modulator," vol. 437, pp. 110-120, 2019.

[15].RG. T. Reed, G. Mashanovich, F. Y. Gardes, and D. J. N. p. Thomson, "Silicon optical modulators," vol. 4, no. 8, p. 518, 2010.

[16].P. O. Weigel, High-Speed Hybrid Silicon-Lithium Niobate Electro-Optic Modulators \& Related Technologies. University of California, San Diego, 2018.

[17].G. J. W. E. o. T. Keiser, "Optical fiber communications," 2003.

[18]M. Martino et al., "Pulsed laser deposition of active waveguides," Thin Solid Films, vol. 433, no. 1-2, pp. 3944, 2003.

[19].R. Wu et al., "Long low-loss-litium niobate on insulator waveguides with sub-nanometer surface roughness," vol. 8, no. 11, p. 910, 2018.

[20].A. Rao et al., "Heterogeneous microring and Mach-Zehnder modulators based on lithium niobate and chalcogenide glasses on silicon," vol. 23, no. 17, pp. 22746-22752, 2015.

[21].E. L. Wooten et al., "A review of lithium niobate modulators for fiber-optic communications systems," vol. 6, no. 1 , pp. 69-82, 2000.

[22].D. Janner, D. Tulli, M. García-Granda, M. Belmonte, V. J. L. Pruneri, and P. Reviews, "Micro-structured integrated electro-optic LiNbO3 modulators," vol. 3, no. 3, pp. 301-313, 2009.

[23].M. He et al., "High-performance hybrid silicon and lithium niobate Mach-Zehnder modulators for 100 Gbit s- 1 and beyond," vol. 13, no. 5, pp. 359-364, 2019.

[24].S. Sederberg, C. J. Firby, S. R. Greig, and A. Y. J. N. Elezzabi, "Integrated nanoplasmonic waveguides for magnetic, nonlinear, and strong-field devices," vol. 6, no. 1, p. 235, 2017.

[25].A. J. Mercante, P. Yao, S. Shi, G. Schneider, J. Murakowski, and D. W. J. O. e. Prather, "110 GHz CMOS compatible thin film LiNbO3 modulator on silicon," vol. 24, no. 14, pp. 15590-15595, 2016.

[26].H. J. C. Jung, "An Integrated Photonic Electric-Field Sensor Utilizing a 1× 2 YBB Mach-Zehnder Interferometric Modulator with a Titanium-Diffused Lithium Niobate Waveguide and a Dipole Patch Antenna," vol. 9, no. 9, p. 459, 2019.

[27]L. Ríos, C. Minor, N. Barboza, and R. J. O. e. Cudney, "Q-switching and quasi-phase-matching using a domain structured LiNbO 3 crystal," vol. 26, no. 13, pp. 17591-17596, 2018.

[28].P. R. Hobson, "Fundamentals of Guided-Wave Optoelectronic Devices, by William SC Chang: Scope: reference. Level: graduate level students studying optoelectronic waveguide devices," ed: Taylor \& Francis, 2011. 